\title{
Severe, but not mild to moderate, non-alcoholic fatty liver disease associated with increased risk of subclinical coronary atherosclerosis
}

\author{
Chia-Chi Hsiao ${ }^{1,2}$, Pai-Hsueh Teng ${ }^{1,2}$, Yun-Ju Wu' ${ }^{1}$, Yi-Wen Shen ${ }^{1}$, Guang-Yuan Mar ${ }^{3}$ and Fu-Zong Wu ${ }^{1,2,4,5^{*}}$
}

\begin{abstract}
Background: Non-alcoholic fatty liver disease (NAFLD) is associated with high risk of cardiovascular disease. The prevalence is increasing to $45-65 \%$ in the general population with routine health check-up, and most subjects have the mild degree NAFLD in recent years. Moreover, there are no studies on the association between NAFLD severity and coronary atherosclerosis in the real-world setting by ultrasonography.

Methods: The aim of this study was to determine the relationship between the severity of NAFLD and subclinical coronary atherosclerosis. Overall, 817 subjects meet criteria for NAFLD were enrolled in the retrospective cohort study (155 subjects were excluded). The severity of NAFLD was divided into the normal, mild, moderate and severe degree based on the finding of abdominal ultrasonography. The assessment of coronary atherosclerosis was based on CAC scan/coronary CT angiography finding in terms of CAC score $\geqq 100$, CAC score $\geqq 400$, CAD-RADS $\geqq 3$ and presence of vulnerable plaque(s).

Results: A significant linear trend was observed between the severity of NAFLD and subclinical coronary atherosclerosis. Compared with the reference group (including normal, mild, and moderate NAFLD), severe degree NAFLD was the independently associated risk of subclinical coronary atherosclerosis in term of CAC score $\geqq 100$, CAC score $\geqq$ 400 , CAD-RADS $\geqq 3$ and presence of vulnerable plaque(s) based on binary logistic regression after adjustment for FRS score and body fat percentage.
\end{abstract}

Conclusions: Severe degree, but not mild to moderate, was associated with high risk of subclinical coronary atherosclerosis, independently of FRS score and body-fat percentage.

Keywords: Fatty liver, Coronary artery atherosclerosis, Vulnerable plaque(s)

\section{Introduction}

Non-alcoholic fatty liver disease (NAFLD) is generally considered as a very common disease in the worldwide at this time. The prevalence of NAFLD in the general

\footnotetext{
*Correspondence: cmvwu1029@gmail.com

1 Section of Thoracic and Circulation Imaging, Department of Radiology,

Kaohsiung Veterans General Hospital, No. 386, Ta-Chung 1st Road,

Kaohsiung 81362, Taiwan

Full list of author information is available at the end of the article
}

population is about $25 \%$ in the world, including Western and Asian countries according to recent literature reviews $[1,2]$. NAFLD comprises a wide range of different conditions from non-alcoholic fatty liver, non-alcoholic steatohepatitis (NASH) to cirrhosis, and the prevalence is increasing worldwide over time due to eating habits and environment change[3, 4]. NAFLD is highly associated with metabolic syndrome and hepatic insulin resistance, which are considered to play an important role in 
developing coronary atherosclerosis [5-7]. Recent systemic review/meta-analyses have investigated the association between presence of NAFLD with coronary arteries disease in terms of obstructive coronary artery disease and coronary artery calcification [8-10]. However, there was limited data regarding the relationship between the severity of NAFLD and subclinical coronary artery disease $[11,12]$. Therefore, the aim of this study was to determine the relationship between subclinical coronary artery disease and the severity of NAFLD in an Asian population in the real-world setting.

\section{Methods}

\section{Clinical, anthropometric and laboratory measurements of study cohort}

The study protocol was approved by the Institutional Review Board Committee of Kaohsiung Veterans General Hospital, Kaohsiung, Taiwan as No. VGHKS 19-CT6-02. The requirement for informed consent was waived due to retrospective study design by the ethics committee of Kaohsiung Veterans General Hospital. All methods were performed in accordance with the relevant guidelines and regulations. In this retrospective study, we reviewed the records of 972 subjects who underwent routine health checkups, abdominal sonogram and coronary $\mathrm{CT}$ angiography examination at the same time in Kaohsiung Veterans General Hospital from January 1, 2018 to December 31, 2018. The inclusion criteria were as follows: (1) all subjects underwent abdominal sonogram and coronary $\mathrm{CT}$ angiography examination at the same time; (2) all subjects are asymptomatic. 155 subjects with at least one of the following: (1) a history of $\geq 10 \mathrm{~g}$ of daily alcohol consumption; (2) liver cirrhosis (defined by ultrasonographic criteria); (3) chronic hepatitis B or C (defined by history, serum hepatitis $B$ surface antigen, and anti-hepatitis $\mathrm{C}$ antibodies) were excluded to meet the diagnostic criteria of NAFLD according to the National Health and Nutrition Examination Survey III criteria [13]. Of the remaining 817 subjects were enrolled into the study population for further analysis show in Fig. 1. Obtained subjects' demographics, cardiovascular risk factors and anthropometric measurements were recorded from referral visit information in the electronic medical records (EMR) system, including age, gender, body mass index (BMI), hypertension, systolic blood pressure, hypertension medication, diabetes mellitus, current smoking habits, the amount of pack-year, waist circumstance, body-fat percentage, and alcohol intake (number, frequency, and alcohol percentage of drinks per week according to the National Institute on Alcohol Abuse and Alcoholism) were recorded for all participants. Anthropometric measurements such as BMI and body-fat percentage were measured using the electric impedance method analyzer (XSCAN PLUS II; Jawon Medical, Gyeongsan-si, South Korea) with the patients minimally clothed and wearing no socks as the previous study described [14]. Complete biochemical and blood examinations including blood levels of low density lipoprotein-cholesterol (LDL-C), high density lipoprotein-cholesterol (HDL-C), triglyceride (TG), total cholesterol, estimated glomerular filtration rate (eGFR), hemoglobin A1c (HbA1c) concentration were also recorded at the same time. Framingham risk score (FRS) percentage was calculated based on the six coronary risk factors including age, gender, total cholesterol, HDL-C, systolic blood pressure, and smoking habits for conventional cardiovascular risk stratification[15].

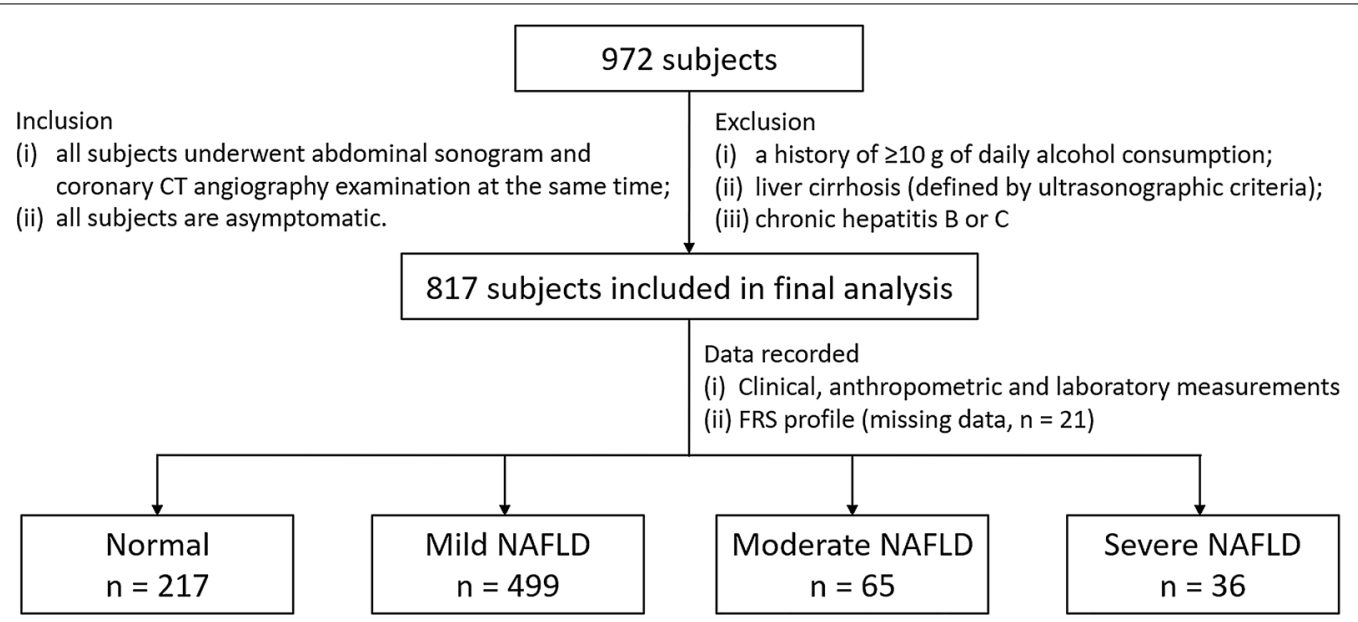

Fig. 1 The flowchart of enrollment and inclusion/exclusion criteria for the study subjects 


\section{Abdominal ultrasound for NAFLD severity assessment} The diagnosis of severity of NAFLD was based on the ultrasonographic findings established according to the practice guideline of the American Gastroenterology Association [16]. All ultrasonographic studies were performed by two imaging units (GE Logic E9, GE Healthcare, Wauwatosa, WI, USA; Acuson S2000, Siemens, Germany) with a 3.5- to $5-\mathrm{MHz}$ convex probe. The severity of NAFLD was divided into the normal, mild, moderate, and severe degree based on hepatic ultrasonography. Mild fatty liver was defined as slightly increased liver parenchyma echogenicity compared to kidney/spleen with normal visualization of portal vein wall and diaphragm. Moderate fatty liver was defined as the moderate increase of liver parenchyma echogenicity compared to kidney/spleen with mild dimness of portal wall and diaphragm. Severe fatty liver was defined as the high increase of liver parenchyma echogenicity compared to kidney/spleen with poor visualization of portal wall and diaphragm due to elevation of echogenicity [17]. All images were retrospectively reviewed by one single expert radiologist with 10 year experience in ultrasonography. The expert was blinded to the clinical and laboratory data of subjects and unaware of the previously study reported.

\section{Coronary artery calcium (CAC) scan and coronary CT angiography}

All enrolled study subjects underwent initial CAC scans and sequential coronary $\mathrm{CT}$ angiography examinations at the same time with 256-slice CT scanner (Revolution CT, GE Healthcare, Milwaukee, USA). We administered oral beta-blockers (metoprolol) if the heart rate exceeded 65 beats per minute an hour before the exams began to start. All subjects received $0.8 \mathrm{mg}$ of sublingual nitroglycerin shortly prior to the CAC scan. The images were analyzed using a dedicated workstation (AW Server, version 3.2, GE Healthcare). All coronary CT angiography examinations were read by two experienced cardiovascular radiologists. Imaging findings of coronary artery stenosis including luminal stenosis severity, plaque composition, and vulnerable plaque(s) characteristics were analyzed according to Coronary Artery Diseases Reporting and Data System (CAD-RADS) classification by 2016 SCCT guideline, including twenty-five coronary segments on the basis of the percentage of cross-sectional area stenosis [18] shown in Additional file 1: Table S1. For CAC score measurements, noncontrast ECG-gated cardiac CT scans were performed based on the Agatston method by using a commercially available CT workstation (AW Server, version 3.2, GE Healthcare). CAC scores were categorized as 4 categories according to the degree of calcification (0-10: minimal; 10-100: mild; 100-400: moderate; $>400$ : severe) [19-21]. In this study cohort, primary endpoints including CAC score $\geqq 100$, CAC score $\geqq 400$, CAD-RADS $\geqq 3$ and presence of vulnerable plaque(s) were assessed for severity of NAFLD associated with subclinical coronary atherosclerosis. The definition of CAD-RADS $\geq 3$ was that presence of significant luminal stenosis $50-69 \% \%$ in any one of the triple coronary trees. There are four main vulnerable plaque features on coronary CT angiography: (1) positive remodeling (2) low attenuation plaque (3) napkin-ring sign (4) spotty calcium. Presence of at least two high-risk plaque feature (s) can be indicated with the definition of vulnerable plaque (s) (Additional file 1: Table S1).

Subclinical coronary atherosclerosis was defined as the presence of atherosclerotic plaque or coronary artery calcification score $(\mathrm{CACS}) \geq 1$ in asymptotic subjects.

\section{Statistical method and analysis}

The patient clinical characteristics, anthropometric and laboratory measurements are expressed as mean \pm standard deviation (SD) or median (interquartile range, IQR) and frequency (\%) for group comparison. Multiple group comparisons were done by analysis of variance (ANOVA) for normally distributed data and by Kruskal-Wallis test for skewed data. We used the post-hoc Bonferroni test to analyze the differences among these four groups. To assess linear trends in the percentage of subclinical atherosclerosis for increasing severity of NAFLD, the general linear model was used to test the linear trend. In the binary logistic regression, the group with normal, mild, and moderate NAFLD was chosen as reference to investigate the relationship between NAFLD severity and coronary atherosclerosis in term of CAC $\geqq 100, C A C \geqq 400$, CAD-RADS $\geqq 3$, and presence of vulnerable plaque(s) after adjustment for FRS score and body fat percentage. A $p$ value $<0.05$ was considered statistically significant.

\section{Results}

Baseline clinical characteristics of the study participants We included 817 subjects (mean age $54.20 \pm 10.02$ years, $60.20 \%$ male) with detailed baseline characteristics and anthropometric and laboratory measurements demonstrated. Among 817 study subjects, 600 (73.43\%) subjects have different degrees of NAFLD. The baseline characteristics, anthropometric and laboratory measurements of the study cohort subjects $(\mathrm{N}=817)$ classified into 4 groups according to the NAFLD severity are shown in Table 1 . There were statistically significant differences across the four groups for the in the clinical characteristics, anthropometric and laboratory measurements and coronary artery finding demonstrated by one-way ANOVA, except for current smoking habits, 


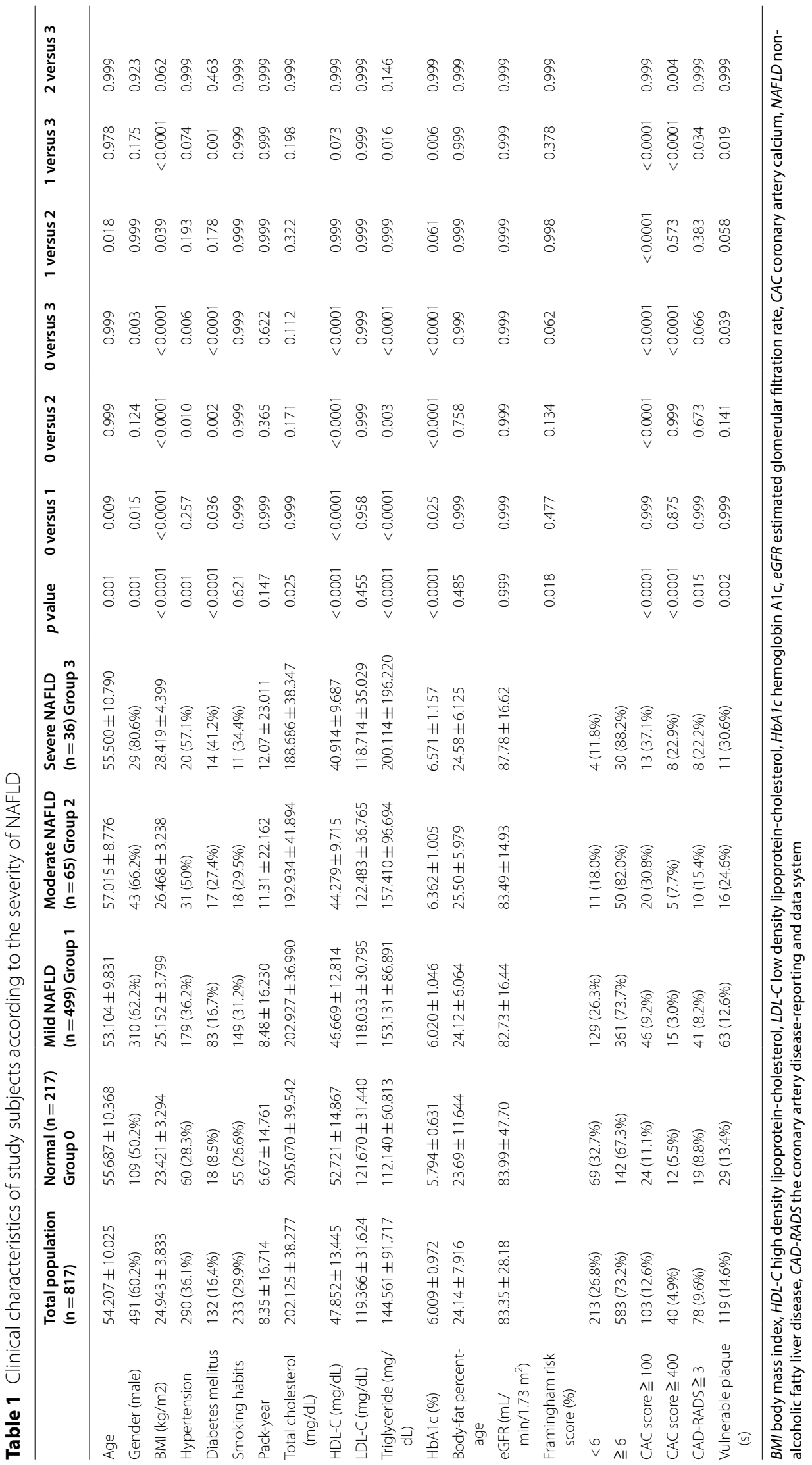


the amount of pack-year, body-fat percentage, eGFR and LDL-C level. In terms of Bonferroni post-hoc tests (intergroup comparison) are also shown in Table 1.

\section{Correlations between severity of NAFLD and subclinical coronary atherosclerosis}

As shown in Fig. 2, subclinical coronary atherosclerosis in term of $C A C \geqq 100, C A C \geqq 400, C A D-R A D S \geqq$ 3 and presence of vulnerable plaques $(+)$ significantly increased as the severity of NAFLD increased by using the general linear model for trend analysis $(C A C \geqq 100, p$ for trend $<0.0001$; $C A C \geqq 400, p$ for trend $=0.003$; CADRADS $\geqq 3, p=0.015$; presence of vulnerable plaques, $p$ for trend $=0.004$.

\section{Severity of NAFLD associated with subclinical coronary atherosclerosis}

Figure 2 shows that the primary endpoints about subclinical coronary atherosclerosis significantly increased as the severity of NAFLD increased by using the general linear model for trend analysis. In addition, posthoc analysis revealed no significant difference in group 1(mild NAFLD) versus group 2 (moderate NAFLD) and group 0 (normal) versus group 1 (mild NAFLD) in terms of subclinical coronary atherosclerosis. Therefore, in the binary logistic regression, the reference group (including normal, mild NAFLD, moderate NAFLD groups) was chosen as the reference to investigate the relationship between the severity of NAFLD and subclinical coronary atherosclerosis. Table 2 shows the multivariate logistic regression analysis to determine the predictors of subclinical coronary atherosclerosis in term of CAC $\geqq 100$, CAC $\geqq 400$, CAD-RADS $\geqq 3$ and presence of vulnerable plaque(s) with adjustment for FRS score and body-fat percentage. For investigation the association with CAC $\geqq 100$, severe degree NAFLD is the independent predictor of CAC $\geqq 100$ after adjusting for FRS score and bodyfat percentage (odds ratio of $2.946,95 \%$ CI 1.017-8.530, $p=0.046)$. For investigation the association with CAC $\geqq$ 400 , severe degree NAFLD is the independent predictor of CAC $\geqq 100$ after adjusting for FRS score and body-fat percentage (odds ratio of 4.402, 95\% CI 1.149-16.862, $p=0.031$ ). For investigation the association with CADRADS $\geqq 3$, severe degree NAFLD is the independent predictor of CAD-RADS $\geqq 3$ after adjusting for FRS score and body-fat percentage (odds ratio of 4.091, 95\% CI 1.396-11.993, $p=0.010$ ). For investigation the association with presence of vulnerable plaque(s), severe degree NAFLD is the independent predictor of presence of vulnerable plaque(s) after adjusting for FRS score and
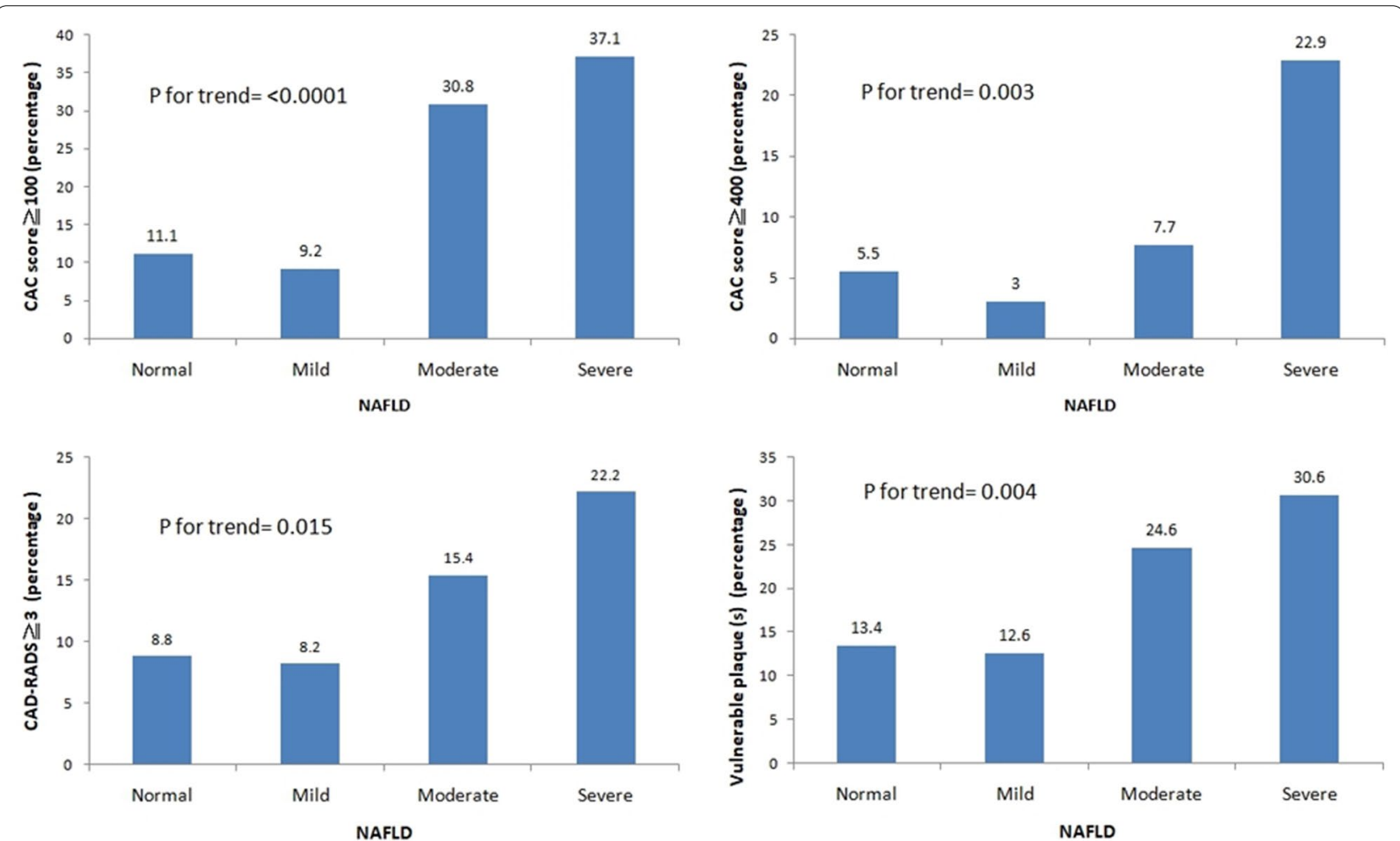

Fig. 2 The linear trend of subclinical coronary atherosclerosis according to severity of NAFLD in term of CAC $\geqq 100, C A C \geqq 400, C A D-R A D S \geqq 3$ and presence of vulnerable plaques $(+)$ 


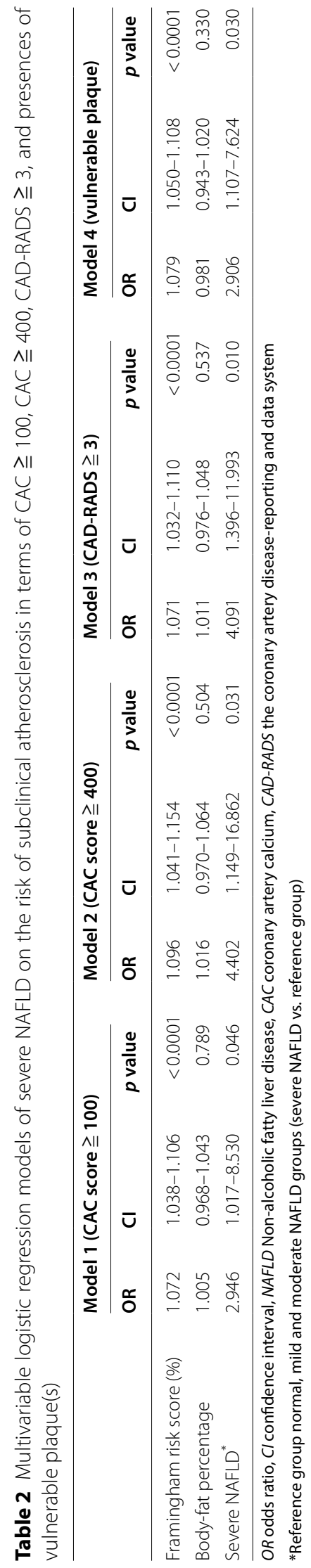


body-fat percentage (odds ratio of 2.906, 95\% CI 1.1077.624, $p=0.030$ ).

\section{Discussion}

The focus of the current study is to investigate the relationship between the severity of NAFLD and subclinical coronary atherosclerosis. In this study, we demonstrated three major findings. The first one is that there was no significant difference in terms of subclinical coronary atherosclerosis between the two groups (normal group versus NAFLD group shown in Additional file 1: Table S2). However, a significant difference in term of subclinical coronary atherosclerosis between the two groups observed (the reference group versus severe NAFLD group shown in Additional file 1: Table S3). Second, a significant linear trend was observed between the severity of NAFLD and subclinical coronary atherosclerosis. Third, for investigation of subclinical coronary atherosclerosis in terms of $C A C \geqq 100, C A C \geqq 400, C A D-R A D S \geqq 3$, and presence of vulnerable plaque(s), severe degree NAFLD is the independent predictor of subclinical coronary atherosclerosis after adjusting for FRS score and body fat percentage.

As for the relationship between NAFLD and cardiovascular disease, previous studies including systemic review-metaanalysis have demonstrated that a significant association between NAFLD and subclinical coronary atherosclerosis, obstructive CAD and major adverse cardiovascular events [8-10, 22]. However previous limited studies have investigated the severity degree of NAFLD associated CAD in asymptomatic or symptomatic subjects $[11,12]$. In addition, the prevalence of NAFLD has increased from $25-30 \%$ in 2012 to $45-65 \%$ in 2018 [2, 12 , 23]. Recently, the prevalence of lean NAFLD or overweight/obese-NAFLD has grown increasingly [14, 24]. It has become a significant public health concern due to current westernized diets, lifestyle habits change and obesity epidemic in Asia [25]. The prevalence of NAFLD found in our study population was high, reaching $73.43 \%$. Of the 600 subjects with NAFLD, 499 (83.16\%) had mild NAFLD, 65 (10.83\%) had moderate NAFLD, and 36 (6\%) had severe NAFLD. Most subjects with NAFLD are clinically silent and asymptomatic, except for a variable degree of fatigue. This is in line with previous research that shows the prevalence has increased recently.

Therefore, it is very important to address the impact of the different severity of NAFLD on coronary atherosclerosis in the epidemic area of the wide spectrum of NAFLD. Our study showed that severity degree of NAFLD was positively correlated with subclinical coronary atherosclerosis, especially in severe degree NAFLD. This study provides a novel finding that relationship between mild to moderate NAFLD and subclinical coronary atherosclerosis was not significant; however, a positive significant association was demonstrated between severe NAFLD and subclinical coronary atherosclerosis, especially for obstructive CAD. Our study finding could partially explain that few studies analyzing the relationship between NAFLD and CAD, and they present controversial results, which may be due to different spectrums of NAFLD severity [26]. As for the mechanism underlying the positive relationship between severe NAFLD and CAD, abnormal insulin resistance, inflammation with oxidative stress and endothelial dysfunction may play an important pathway for the development of atherosclerosis with a potential dose-response relationship [5, 6, 27].

It has recently been observed that the eradication of $\mathrm{HCV}$ (hepatitis $\mathrm{C}$ virus) infection with direct-acting antiviral therapy, probably through the elimination of chronic inflammation due to the infection, leads to a reduction in insulin-resistance as well as both the onset of diabetes $[28,29]$. These results indirectly confirm the potential mechanism of insulin resistance in the relationship between NAFLD and atherosclerotic disease.

The study has several limitations. First, this retrospective cohort study was cross-sectional design, which limits its cause-and-effect conclusion between severity of NAFLD and subclinical coronary atherosclerosis. In the severe NAFLD group, $41.2 \%$ of subjects had diabetes. Therefore, this patient setting was mostly affected by insulin resistance with increasing the risk of atherosclerosis.

In addition, we didn't collect the C-reactive protein (CRP) level, medication history (such as statins, antiplatelet agents, and Glucagon-like peptide-1 agonists), insulin-mediated cytokine and coronary segment severity score. Therefore, the underlying mechanism assessing the association of the level of CRP, the severity of NAFLD, and subclinical coronary atherosclerosis could not be addressed in this study [30-36]. Second, our retrospective cohort subjects were self-referred, suggestive of a potential selection bias. Third, there are some shortcomings such as operator-dependent and subjective evaluation of NAFLD by ultrasonography [37]. However, the meta-analysis has demonstrated that ultrasonography allows for reliable and accurate diagnosis of moderate-severe fatty liver [38]. In addition, ultrasonography is a currently low cost, non-invasive and easily feasible diagnostic tool for screening for NAFLD in real-world settings. Fourth, this study cohort is based on the population in Taiwan. Therefore, it is not be generalized to another different racial and ethnic population due to differences in health status, dietary habits, and lifestyle. In addition, our study primary endpoint is to investigate the association of fatty liver with coronary atherosclerosis, 
independently of conventional cardiovascular risks (FRS percentile). The impact of gender difference would not be investigated due to relative small sample size in the severe NAFLD group. Fifth, previous studies/guidelines did not recommend cardiac CT angiography as a screening test in asymptomatic low-risk subjects [39, 40]. However, several studies have retrospectively demonstrated that male gender, diabetes mellitus, and smoking amount are predictors of subclinical coronary atherosclerosis or noncalcified plaque, even in young adults or health checkup populations [41-44]. Our study used a similar retrospective cohort design to investigate the association between the degree of fatty liver and coronary atherosclerosis, independently of traditional cardiovascular risk factors (FRS percentile). However, there are still a small number of missing values existed in FRS profile $(\mathrm{N}=21)$. Finally, this study tried to investigate the relationship between fatty liver and coronary atherosclerosis. Future studies are warranted to investigate the prediction model including conventional cardiovascular risk factors and severity of NAFLD for coronary atherosclerosis prediction [45].

\section{Conclusion}

In conclusion, our results indicate that severity of NAFLD is closely associated with subclinical coronary atherosclerosis, as assessed by coronary CTA and CAC scan, regardless of FRS score and body-fat percentage. This real-world work provides evidence that severe degree, but not mild to moderate NAFLD associated high risk of obstructive CAD. Accordingly, we recommend that physicians should actively care for subjects with the severe degree NAFLD and offer information about high risk of coronary atherosclerosis in the real-world ultrasound health-checkup setting. In the future, the prediction model including conventional cardiovascular risk factors and severity of NAFLD warranted further investigation.

\section{Supplementary Information}

The online version contains supplementary material available at https://doi. org/10.1186/s12872-021-02060-z.

Additional file 1: Supplement Table 1. Definition of CAD-RADS category system and vulnerable plaque(s). Supplement Table 2. Clinical characteristics of study subjects according to the severity of with or without NAFLD. Supplement Table 3. Clinical characteristics of study subjects according to the reference group and severe NAFLD group

\section{Acknowledgements}

Not applicable.

\section{Authors' contributions}

Conceptualization, FZW; data curation, $\mathrm{CCH}$ and PHT; formal analysis, FZW and YJW; funding acquisition, FZW; methodology, YWS and GYM; writing — draft editing, CCH, FZW, and YJW; All authors have read and agreed to the published version of the manuscript.
Funding

This study was supported by a Grant from Kaohsiung Veterans General Hospital, VGHKS108-159, VGHKS109-147, MOST 108-2314-B-075B-008, MOST 109-2314-B-075B-006-, Taiwan, R.O.C.

\section{Availability of data and materials}

The datasets used and/or analyzed during the current study are available from the corresponding author on reasonable request.

\section{Declarations}

\section{Ethics approval and consent to participate}

The study protocol was approved by the Institutional Review Board Committee of Kaohsiung Veterans General Hospital, Kaohsiung, Taiwan as No. VGHKS 19-CT6-02. Participants' informed consent were waived by the ethics committee of Kaohsiung Veterans General Hospital, and were assessed in accordance with the Helsinki declaration.

\section{Consent for publication}

Not applicable.

\section{Competing interests}

The authors declare no conflicts of interest.

\section{Author details}

${ }^{1}$ Section of Thoracic and Circulation Imaging, Department of Radiology, Kaohsiung Veterans General Hospital, No. 386, Ta-Chung 1st Road, Kaohsiung 81362, Taiwan. ${ }^{2}$ Department of Medical Imaging and Radiology, Shu-Zen Junior College of Medicine and Management, Kaohsiung, Taiwan. ${ }^{3}$ Physical Examination Center, Kaohsiung Veterans General Hospital, Kaohsiung, Taiwan. ${ }^{4}$ Faculty of Medicine, School of Medicine, National Yang-Ming University, Taipei, Taiwan. ${ }^{5}$ Department of Medical Education and Research, Kaohsiung Veterans General Hospital, Kaohsiung, Taiwan.

Received: 26 March 2021 Accepted: 12 May 2021

Published online: 19 May 2021

\section{References}

1. Araújo AR, Rosso N, Bedogni G, Tiribelli C, Bellentani S. Global epidemiology of non-alcoholic fatty liver disease/non-alcoholic steatohepatitis: What we need in the future. Liver Int. 2018;38(S1):47-51.

2. Younossi ZM. Non-alcoholic fatty liver disease \&\#x2013; A global public health perspective. J Hepatol. 2019;70(3):531-44.

3. Mitra S, De A, Chowdhury A. Epidemiology of non-alcoholic and alcoholic fatty liver diseases. Transl Gastroenterol Hepatol. 2020;5:16-16.

4. Andronescu Cl, Purcarea MR, Babes PA. Nonalcoholic fatty liver disease: epidemiology, pathogenesis and therapeutic implications. J Med Life. 2018;11(1):20-3.

5. Lonardo A, Ballestri S, Marchesini G, Angulo P, Loria P. Nonalcoholic fatty liver disease: a precursor of the metabolic syndrome. Dig Liver Dis. 2015:47(3):181-90.

6. Abdallah LR, de Matos RC, e Souza YPDM, Vieira-Soares D, MullerMachado G, Pollo-Flores P. Non-alcoholic Fatty Liver Disease and Its Links with Inflammation and Atherosclerosis. Curr Atheroscler Rep. 2020;22(1):7.

7. Wu F-Z, Chou K-J, Huang Y-L, Wu M-T. The relation of location-specific epicardial adipose tissue thickness and obstructive coronary artery disease: systemic review and meta-analysis of observational studies. BMC Cardiovasc Disord. 2014;14:62-62.

8. Lu H, Liu H, Hu F, Zou L, Luo S, Sun L. Independent association between nonalcoholic fatty liver disease and cardiovascular disease: a systematic review and meta-analysis. Int J Endocrinol. 2013;2013:124958-124958.

9. Jaruvongvanich V, Wirunsawanya K, Sanguankeo A, Upala S. Nonalcoholic fatty liver disease is associated with coronary artery calcification: a systematic review and meta-analysis. Dig Liver Dis. 2016;48(12):1410-7.

10. Ampuero J, Gallego-Durán R, Romero-Gómez M. Association of NAFLD with subclinical atherosclerosis and coronary-artery disease: meta-analysis. Rev Esp Enferm Dig. 2015;107(1):10-6. 
11. Inci MF, Özkan F, Ark B, Vurdem ÜE, Ege MR, Sincer I, Zorlu A. Sonographic evaluation for predicting the presence and severity of coronary artery disease. Ultrasound Q. 2013;29(2):125-30.

12. Lee JE, Lee YJ, Chung SY, Cho HW, Park BJ, Jung DH. Severity of nonalcoholic fatty liver disease is associated with subclinical cerebro-cardiovascular atherosclerosis risk in Korean men. PLOS ONE. 2018;13(3):e0193191-e0193191.

13. Lazo M, Hernaez R, Eberhardt MS, Bonekamp S, Kamel I, Guallar E, Koteish A, Brancati FL, Clark JM. Prevalence of nonalcoholic fatty liver disease in the United States: the Third National Health and Nutrition Examination Survey, 1988-1994. Am J Epidemiol. 2013;178(1):38-45.

14. Hsu C-L, Wu F-Z, Lin K-H, Chen Y-H, Wu P-C, Chen Y-H, Chen C-S, Wang $\mathrm{W}-\mathrm{H}, \mathrm{Mar} \mathrm{G}-\mathrm{Y}, \mathrm{Yu} \mathrm{H}-\mathrm{C}$. Role of fatty liver index and metabolic factors in the prediction of nonalcoholic fatty liver disease in a lean population receiving health checkup. Clin Transl Gastroenterol. 2019;10(5):1-8.

15. Sohn C, Kim J, Bae W. The framingham risk score, diet, and inflammatory markers in Korean men with metabolic syndrome. Nutr Res Pract. 2012;6(3):246-53

16. Chalasani N, Younossi Z, Lavine JE, Diehl AM, Brunt EM, Cusi K, Charlton M, Sanyal AJ. The diagnosis and management of non-alcoholic fatty liver disease: practice guideline by the American Association for the Study of Liver Diseases, American College of Gastroenterology, and the American Gastroenterological Association. Hepatology. 2012;55(6):2005-23.

17. Saadeh $S$, Younossi ZM, Remer EM, Gramlich T, Ong JP, Hurley M, Mullen KD, Cooper JN, Sheridan MJ. The utility of radiological imaging in nonalcoholic fatty liver disease. Gastroenterology. 2002;123(3):745-50.

18. Cury RC, Abbara S, Achenbach S, Agatston A, Berman DS, Budoff MJ, Dill $\mathrm{KE}$, Jacobs JE, Maroules CD, Rubin GD, et al. Coronary Artery Disease Reporting and Data System (CAD-RADS): an Expert Consensus Document of SCCT, ACR and NASCl: endorsed by the ACC. JACC Cardiovasc Imaging. 2016;9(9):1099-113.

19. Rumberger JA, Brundage BH, Rader DJ, Kondos G. Electron beam computed tomographic coronary calcium scanning: a review and guidelines for use in asymptomatic persons. Mayo Clin Proc. 1999;74(3):243-52.

20. Neves PO, Andrade J, Monção H. Coronary artery calcium score: current status. Radiol Bras. 2017;50(3):182-9.

21. Shen YW, Wu YJ, Hung YC, Hsiao CC, Chan SH, Mar GY, Wu MT, Wu FZ. Natural course of coronary artery calcium progression in Asian population with an initial score of zero. BMC Cardiovasc Disord. 2020;20(1):212.

22. Liu Y, Zhong G-C, Tan H-Y, Hao F-B, Hu J-J. Nonalcoholic fatty liver disease and mortality from all causes, cardiovascular disease, and cancer: a metaanalysis. Sci Rep. 2019;9(1):11124.

23. Mahale AR, Prabhu SD, Nachiappan M, Fernandes M, Ullal S. Clinical relevance of reporting fatty liver on ultrasound in asymptomatic patients during routine health checkups. J Int Med Res. 2018;46(11):4447-54.

24. Denkmayr L, Feldman A, Stechemesser L, Eder SK, Zandanell S, Schranz M, Strasser M, Huber-Schönauer U, Buch S, Hampe J, et al. Lean patients with non-alcoholic fatty liver disease have a severe histological phenotype similar to obese patients. J Clin Med. 2018;7(12):562.

25. Yasutake K, Kohjima M, Kotoh K, Nakashima M, Nakamuta M, Enjoji M. Dietary habits and behaviors associated with nonalcoholic fatty liver disease. World J Gastroenterol. 2014;20(7):1756-67.

26. Yilmaz Y, Senates E, Ayyildiz T, Colak Y, Tuncer I, Ovunc AOK, Dolar E, Kalayci C. Characterization of nonalcoholic fatty liver disease unrelated to the metabolic syndrome. Eur J Clin Invest. 2012;42(4):411-8.

27. Niikura T, Imajo K, Ozaki A, Kobayashi T, Iwaki M, Honda Y, Kessoku T, Ogawa $Y$, Yoneda M, Kirikoshi $\mathrm{H}$, et al. Coronary artery disease is more severe in patients with non-alcoholic steatohepatitis than fatty liver. Diagnostics (Basel). 2020;10(3):129.

28. Adinolfi LE, Petta S, Fracanzani AL, Coppola C, Narciso V, Nevola R, Rinaldi L, Calvaruso V, Staiano L, Di Marco V, et al. Impact of hepatitis C virus clearance by direct-acting antiviral treatment on the incidence of major cardiovascular events: a prospective multicentre study. Atherosclerosis. 2020:296:40-7.

29. Adinolfi LE, Petta S, Fracanzani AL, Nevola R, Coppola C, Narciso V, Rinaldi $L$, Calvaruso V, Pafundi PC, Lombardi R, et al. Reduced incidence of type 2 diabetes in patients with chronic hepatitis $C$ virus infection cleared by direct-acting antiviral therapy: a prospective study. Diabetes Obes Metab. 2020;22(12):2408-16.
30. Cozzolino D, Sessa G, Salvatore T, Sasso FC, Giugliano D, Lefebvre PJ, Torella R. The involvement of the opioid system in human obesity: a study in normal weight relatives of obese people. J Clin Endocrinol Metab. 1996;81(2):713-8.

31. Esposito K, Ciotola M, Sasso FC, Cozzolino D, Saccomanno F, Assaloni R, Ceriello A, Giugliano D. Effect of a single high-fat meal on endothelial function in patients with the metabolic syndrome: role of tumor necrosis factor-alpha. Nutr Metab Cardiovasc Dis. 2007;17(4):274-9.

32. Marfella R. D’Amico M, Di Filippo C, Siniscalchi M, Sasso FC, Ferraraccio F, Rossi F, Paolisso G: The possible role of the ubiquitin proteasome system in the development of atherosclerosis in diabetes. Cardiovasc Diabetol. 2007:6:35-35.

33. Marfella R, Ferraraccio F, Rizzo MR, Portoghese M, Barbieri M, Basilio C, Nersita R, Siniscalchi LI, Sasso FC, Ambrosino I, et al. Innate immune activity in plaque of patients with untreated and L-thyroxine-treated subclinical hypothyroidism. J Clin Endocrinol Metab. 2011;96(4):1015-20.

34. Torella D, Ellison GM, Torella M, Vicinanza C, Aquila I, laconetti C, Scalise $\mathrm{M}$, Marino $\mathrm{F}$, Henning $\mathrm{BJ}$, Lewis FC, et al. Carbonic anhydrase activation is associated with worsened pathological remodeling in human ischemic diabetic cardiomyopathy. J Am Heart Assoc. 2014;3(2):e000434.

35. Minutolo R, Gabbai FB, Provenzano M, Chiodini P, Borrelli S, Garofalo C, Sasso FC, Santoro D, Bellizzi V, Conte G, et al. Cardiorenal prognosis by residual proteinuria level in diabetic chronic kidney disease: pooled analysis of four cohort studies. Nephrol Dial Transplant. 2018;33(11):1942-9.

36. Sasso FC, Pafundi PC, Marfella R, Calabrò P, Piscione F, Furbatto F, Esposito G, Galiero R, Gragnano F, Rinaldi L, et al. Adiponectin and insulin resistance are related to restenosis and overall new $\mathrm{PCl}$ in subjects with normal glucose tolerance: the prospective AIRE Study. Cardiovasc Diabetol. 2019:18(1):24.

37. Cengiz M, Sentürk S, Cetin B, Bayrak AH, Bilek SU. Sonographic assessment of fatty liver: intraobserver and interobserver variability. Int J Clin Exp Med. 2014;7(12):5453-60.

38. Hernaez R, Lazo M, Bonekamp S, Kamel I, Brancati FL, Guallar E, Clark JM. Diagnostic accuracy and reliability of ultrasonography for the detection of fatty liver: a meta-analysis. Hepatology (Baltimore, MD). 2011:54(3):1082-90.

39. van der Wall EE. Screening in asymptomatic coronary artery disease: helpful, redundant or harmful? Neth Hear J. 2014;22(12):531-2.

40. Taylor AJ, Cerqueira M, Hodgson JM, Mark D, Min J, O'Gara P, Rubin GD. ACCF/SCCT/ACR/AHA/ASE/ASNC/NASCI/SCAI/SCMR 2010 appropriate use criteria for cardiac computed tomography. Circulation. 2010;122(21):e525-55.

41. Jin KN, Chun EJ, Lee CH, Kim JA, Lee MS, Choi SI. Subclinical coronary atherosclerosis in young adults: prevalence, characteristics, predictors with coronary computed tomography angiography. Int J Cardiovasc Imaging. 2012;28(Suppl 2):93-100.

42. Noh DW, Kim S. Associations between coronary artery stenosis detected by coronary computed tomography angiography and the characteristics of health checkup examinees in the Republic of Korea. Radiography (Lond). 2020;26(1):22-6.

43. Plank F, Friedrich G, DichtI W, Klauser A, Jaschke W, Franz WM, Feuchtner $\mathrm{G}$. The diagnostic and prognostic value of coronary CT angiography in asymptomatic high-risk patients: a cohort study. Open Heart. 2014; 1 (1):e000096.

44. Han D, Hartaigh B, Gransar H, Lee JH, Rizvi A, Baskaran L, Schulman-Marcus J, Dunning A, Achenbach S, Al-Mallah MH, et al. Incremental prognostic value of coronary computed tomography angiography over coronary calcium scoring for major adverse cardiac events in elderly asymptomatic individuals. Eur Heart J Cardiovasc Imaging. 2018;19(6):675-83.

45. Wu Y-J, Mar G-Y, Wu M-T, Wu F-Z. A LASSO-derived risk model for subclinical CAC progression in an Asian population with an initial score of zero. Front Cardiovasc Med. 2021;7(385):1-10.

\section{Publisher's Note}

Springer Nature remains neutral with regard to jurisdictional claims in published maps and institutional affiliations. 